\title{
Localization of Two-Component Bose-Einstein Condensates in Optical Lattices
}

\author{
Elena A. Ostrovskaya and Yuri S. Kivshar \\ Nonlinear Physics Group, ARC Centre for Quantum-Atom Optics and Research School of Physical Sciences and Engineering, \\ Australian National University, Canberra ACT 0200, Australia
}

(Dated: October 30, 2018)

\begin{abstract}
We reveal underlying principles of nonlinear localization of a two-component Bose-Einstein condensate loaded into a one-dimensional optical lattice. Our theory shows that spin-dependent optical lattices can be used to manipulate both the type and magnitude of nonlinear interaction between the ultracold atomic species and to observe nontrivial two-componentnlocalized states of a condensate in both bands and gaps of the matter-wave band-gap structure.
\end{abstract}

Recent experimental studies of Bose-Einstein condensates (BECs) in optical lattices, including Bloch-band spectroscopy [1], patterned loading of the lattice [2], and nonlinear tunnelling [3] , demonstrate unprecedented levels of manipulation of the coherent matter waves. Tunability of the periodic lattice potential combined with intrinsic nonlinearity of BEC provides an excellent testbed for many concepts of condensed matter physics and nonlinear physics. In particular, strong parallels have been drawn [4] between nonlinear optics of ultracold atoms in optical lattices and behaviour of optical waves in periodic nonlinear media, where instability and localization phenomena have been well explored [5]. Akin to optical structures with periodic refractive index, such as waveguide arrays and photonic crystals, optical lattices form a band-gap structure for coherent matter waves which modify diffraction properties of atomic wavepackets [6]. The coexistence of both normal and anomalous diffraction regimes was predicted to lead to a nonlinear localization of condensate with both attractive and repulsive interactions in the gaps of a linear Bloch-wave spectrum 7]. Recent experiments on diffraction management of BEC in lattices [8, 9] have paved the way to the first observation of a localized atomic wavepacket (bright gap soliton) in a BEC with repulsive interactions 10.

Most of the theoretical and experimental studies of coherent matter waves in optical lattices are concerned with single-component BECs. However, it is well known that condensate mixtures display many novel physical effects not found in single-species BECs 11], including domain separation of the ground state and stabilization of nonlinear localized states. Experiments on spin-dependent optical lattices [12] have shown that a two-component BEC composed of two hyperfine states of the same atomic species can be effectively and coherently manipulated in an optical lattice. Recently, analysis of periodic (Bloch) states of a multi-component BEC in a lattice 13, 14 has revealed that the modulational instability of excited periodic states can potentially lead to the formation of multicomponent localized states (solitons) 14]. This conclusion agrees with the recent theoretical study of nonlinear inter-gap localization of multi-component coherent light fields in periodic optical structures [15].

In this Letter, we address the problem of nonlinear localization of a two-component condensate and formation of two-component matter-wave solitons in an optical lattice. We show that an optical lattice can be used to modify the type of the effective nonlinear interactions both within and between the condensate species, without the Feshbach resonance manipulation of the scattering lengths. As a consequence, novel types of nonlinear localization of coherent matter waves can be achieved both in gaps and bands of the linear Bloch-wave spectrum. In particular, when one of the condensate components is in the periodic Bloch state, the second component exhibits effective periodic potential combined with the optical potential and that induced by the mean-field of the Bloch wave. As a result, nontrivial localization of a twocomponent BEC in a self-induced lattice can occur in the form of a two-component "dark-bright" band-gap soliton. The effects of two-component localization discussed here can be explored in the current BEC experiments, as well as in the optically-induced photonic lattices [5].

We model the dynamics of a two-component BEC with repulsive interactions in an optical lattice by the meanfield equations for the wavefunctions of the condensate components $|a\rangle$ and $|b\rangle$ :

$$
i \frac{\partial}{\partial t}\left(\begin{array}{c}
\Psi_{a} \\
\Psi_{b}
\end{array}\right)=\left(\begin{array}{cc}
\hat{L}_{a} & \hat{L}_{a b} \\
\hat{L}_{a b}^{*} & \hat{L}_{b}
\end{array}\right)\left(\begin{array}{c}
\Psi_{a} \\
\Psi_{b}
\end{array}\right)
$$

where $\hat{L}_{n}=-\partial^{2} / \partial x^{2}+V_{n}(x)+g_{n n}\left|\Psi_{n}\right|^{2}$, and $\hat{L}_{a b}=$ $g_{a b} \Psi_{a} \Psi_{b}^{*}$. Here we assume that the two BEC components can exhibit different potentials, namely: $V_{a}(x)=$ $V_{0} \sin ^{2}\left(k_{L} x\right)$ and $V_{b}(x)=V_{0} \sin ^{2}\left(k_{L} x+\theta\right)$, which can be shifted relative to each other, e.g. by varying the polarization angle in the case of a spin-dependent lattice [12]. These potentials are less constrained than those in 12 to allow for consideration of a general setup where the condensate components may actually belong to different atomic species 16], being manipulated independently. The one-dimensional model (10) is derived for strongly anisotropic BEC clouds with the tight confinement direction transverse to that of the lattice [7] and made dimensionless by adopting the lattice units of length $x_{0}=k_{L}^{-1}$ (typically $\sim 10^{-1} \mu \mathrm{m}$ ) and energy $E_{r}=\hbar^{2} k_{L}^{2} / 2 m$. The stationary states of the wavefunction of the $n$-th BEC component are found as $\Psi_{n}(x, t)=\psi_{n}(x) \exp \left(-i \mu_{n} t\right)$, 

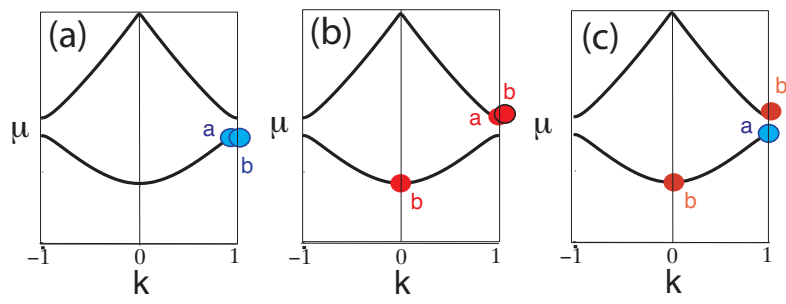

FIG. 1: Chemical potential and momentum of the BEC components, relative to the lattice band-gap structure, necessary to achieve (a) attractive-attractive, (b) repulsive-repulsive, and (c) repulsive-attractive effective nonlinear interactions. For the fixed parameters of the $|a\rangle$ component either of the $|b\rangle$ locations can be chosen. Colors code different signs of effective diffraction.

where $\mu_{n}$ is the chemical potential normalized by $E_{r}$. Without the repulsive mean-field interactions, the ground and excited states of each of the BEC components are periodic matter waves: $\psi_{n}=\phi_{n}(x) \exp (i k x)$, where the lattice momentum $k$ lies within the Brillouin zone. The periodicity of the lattice leads to the band-gap structure of the spectrum $\mu_{n}(k)$. At the $j$-th edge of the Brillouin zone $\mu_{n} \equiv \mu_{n}^{\{j\}}$, the wavefunction $\phi_{n}^{\{j\}}(x)=$ $\phi_{n}^{\{j\}}\left(x+\pi / k_{L}\right)$ is a Bloch state with the lattice periodicity. Schematics of the Bloch-wave spectrum in the reduced-zone representation $(|k|<1)$ is shown in Fig. 1

A stationary BEC wavepacket located, in the momentum $k$-space, near the $j$-th band edge can be described as a corresponding Bloch wave, dressed by a slowly varying (in $x$ ) envelope, $\Psi_{n}\left(x, t ; \mu_{n}^{\{j\}}\right)=\Phi_{n}(x, t) \phi_{n}^{\{j\}}(x)$. Considering that the condensate components $|a\rangle$ and $|b\rangle$ can be positioned at the band edges $i$ and $j$, respectively, the envelope equations take the form [15]:

$$
\begin{aligned}
& -i \frac{\partial \Phi_{a}}{\partial t}=\left[\frac{1}{2} D_{a}^{\{i\}} \frac{\partial^{2}}{\partial x^{2}}-\tilde{g}_{a a}^{\{i i\}}\left|\Phi_{a}\right|^{2}-\tilde{g}_{a b}^{\{i j\}}\left|\Phi_{b}\right|^{2}\right] \Phi_{a} \\
& -i \frac{\partial \Phi_{b}}{\partial t}=\left[\frac{1}{2} D_{b}^{\{j\}} \frac{\partial^{2}}{\partial x^{2}}-\tilde{g}_{b b}^{\{j j\}}\left|\Phi_{b}\right|^{2}-\tilde{g}_{b a}^{\{i j\}}\left|\Phi_{a}\right|^{2}\right] \Phi_{b},
\end{aligned}
$$

where the effective diffraction of the wavepacket is defined by the curvature of the corresponding band edge, $D_{a, b}^{\{i, j\}}=\left(\partial^{2} \mu_{a, b} / \partial k^{2}\right)_{\mu_{a, b}=\mu_{a, b}^{\{i, j\}}}$, and the effective interaction coefficients depend on the symmetries of the Bloch states at the band edges: $\tilde{g}_{a b}^{\{i j\}}=g_{a b}^{\{i j\}} \int\left|\phi_{a}^{\{j\}} \phi_{b}^{\{j\}}\right|^{2} d x$. The relative shift of the spin-dependent lattice potentials can enhance or dampen the cross-species nonlinear interaction without any change in the effective diffraction, which introduces a new degree of experimental control into the system. Eqs. (2) and their complex conjugate form are equivalent, so even for the case of anomalous diffraction $\left(D_{n}<0\right)[\underline{6}]$ the equations can always be rewritten in the more intuitive normal diffraction form, but with opposite signs of the effective nonlinear coefficients.

Envelope equations (2) predict three types of the effective nonlinear interactions that can be realized for two-component BECs in a lattice. These regimes can be achieved by appropriate placing of the BEC wavepackets parameters relative to the band structure, as illustrated schematically in Figs. 11(a-c). Below we consider only the main band $\left(B 1:\left.\mu^{\{1\}}\right|_{k=0} \leq \mu \leq\left.\mu^{\{2\}}\right|_{k=1}\right)$ and the first excited band $\left(B 2:\left.\mu^{\{3\}}\right|_{k=1} \leq \mu \leq \mu=\left.\mu^{\{4\}}\right|_{k=0}\right)$ of the lattice that can be populated in experiments [1].

Attractive-attractive interaction regime [Fig. 1(a)] can be realized when $\tilde{g}_{a a, b b}, \tilde{g}_{a b, b a}<0,\left|\tilde{g}_{a a}\right|=\left|\tilde{g}_{b b}\right|=\left|\tilde{g}_{a b}\right|=$ $\left|\tilde{g}_{b a}\right|$. In this regime, near the band edge $\mu=\left.\mu^{\{2\}}\right|_{k=1}$ both condensate components exhibit anomalous diffraction, and therefore can exhibit self-focusing in the form of two-component bright gap solitons. The envelope equations (2) take the form of the integrable Manakov system, well studied in the context of nonlinear optics (see, e.g., [1]). According to Eq. (2), both localized components can be treated as ground states of the same effective potential; they have equal chemical potentials $\mu_{a}=\mu_{b}$ and widths of the spatial density distribution envelopes: $\Phi_{a}(x)=\Phi_{0}(x) \cos \alpha, \Phi_{b}(x)=\Phi_{0}(x) \sin \alpha$, where $\Phi_{0}(x)=\sqrt{\mu_{a} / \tilde{g}_{a a}} \operatorname{sech}\left(\sqrt{2 \mu_{a}} x\right)$, and $\alpha$ is an arbitrary parameter. The existence domain for twocomponent solutions in the parameter space $\left\{\mu_{a}, \mu_{b}\right\}$ can also be calculated using linear waveguiding principles. Assuming that the densities of the two component are largely dissimilar, one can decouple Eqs. (11) and find, numerically, spatial profiles of single-component bright gap solitons $\psi_{a}\left(x ; \mu_{a}\right)$ in the entire gap $\mu_{a}^{\{2\}} \leq \mu_{a} \leq \mu_{a}^{\{3\}}$. The low-density second component, $\psi_{b}$ can then be found as a ground state (i.e. lowest-order guided mode) of the effective linear potential combined of the lattice potential and the mean-field potential induced by the $|a\rangle$ component. The cut-off values $\mu_{b}\left(\mu_{a}\right)$ for this ground state define the lower boundary of the existence domain for two-component localized states of the condensate, and the upper boundary is $\mu_{b}=\mu_{a}$. The linear waveguiding analysis of the complete model (1) confirms the prediction of the envelope theory that in the degenerate case of equal effective interaction coefficients the existence domain is confined to the line $\mu_{b}=\mu_{a}$ (see Fig. 2). Remarkably, this degeneracy can be lifted by shifting the lattices trapping the two condensate components relative to each other. The shift destroys the equality between the interaction coefficients so that $\tilde{g_{a b}} / \tilde{g}_{a a}>1$ and, according to the envelope theory, yields the new cut-off values for the $\psi_{b}$ modes, $\mu_{b}=\left(\mu_{a} / 4\right)\left(1-\sqrt{1+8 \tilde{g}_{a b} / \tilde{g}_{a a}}\right)^{2}>\mu_{a}$. The boundaries of the significantly expanded existence domain calculated from the guided-mode analysis of the complete model (11) are shown in Fig. 2 by dashed lines; such a dramatic expansion can be achieved by the relatively small shift of $\theta=\pi / 4$.

Repulsive-repulsive regime [Fig. 1(b)] can be achieved when $\tilde{g}_{a a, b b}, \tilde{g}_{a b, b a}>0,\left|\tilde{g}_{a b}\right|=\left|\tilde{g}_{b a}\right|$, and the wavepackets are located either at the same, $\left|\tilde{g}_{a a}\right|=\left|\tilde{g}_{b b}\right|$, or different, $\left|\tilde{g}_{a a}\right| \neq\left|\tilde{g}_{b b}\right|$, band edges. Let us first consider the BEC wavepacket located at the edge of the sec- 

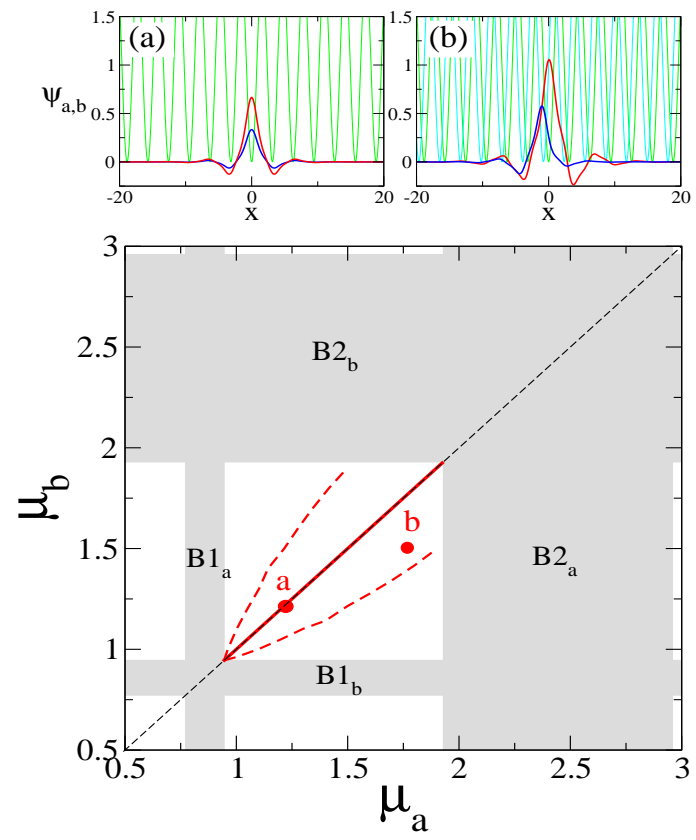

FIG. 2: Existence domain for the bright-bright atomic gap solitons in the $\left\{\mu_{a}, \mu_{b}\right\}$ plane $\left(V_{0}=2, g_{a a}=g_{b b}=g_{a b}=1\right)$. Shaded - two lowest Bloch bands $B 1_{a, b}$ and $B 2_{a, b}$ exhibited by the condensate components $|a\rangle$ and $|b\rangle$. Solid line - existence domain $\mu_{a}=\mu_{b}$ predicted by the envelope theory and linear waveguiding calculations (see text). Dashed lines - borders of the existence domain for the relative lattice shift $\theta=\pi / 4$. Top panels: examples of the condensate wavefunctions at the marked points of the existence domain.

ond band $\left(B 2_{a}\right), \mu_{a}=\left.\mu_{a}^{\{3\}}\right|_{k=1}$. It experiences normal diffraction and can support a dark soliton with the envelope $\Phi_{a}=\sqrt{\mu_{a} / \tilde{g}_{a a}} \tanh \left(\sqrt{\mu_{a a}} x\right)$, imprinted onto the extended background of a Bloch wave $\phi_{a}^{\{3\}}(x)$ [17]. Originating at the band edge, such dark states with zero group velocity can exist within the entire band. Their spatial structure suggests that they can be created experimentally by phase-imprinting technique, which does not require access to the gap. It is easy to see that, in a two-component $\mathrm{BEC}$, the Bloch state in $|a\rangle$-component induces a periodic potential for the $|b\rangle$ component which acts together with the potential of the optical lattice. As a result, the band-gap structure of the Bloch-wave spectrum for the $|b\rangle$-component is significantly modified. The original bands are shifted, so that the edge of the modified second band $\mu_{b}^{\{3\}}$ coincides with the chemical potential of the nonlinear Bloch state $\phi_{a}^{\{3\}}$ (see Fig. 3). The remarkable effect of this condensate-induced lattice potential is that the $|b\rangle$-component can now be spatially localized in every gap of the induced band-gap structure in the form of a bright gap soliton. Moreover, the existence domains for the coupled states calculated using the waveguiding properties of the dark in-band solitons, lie entirely within the original bands $B 1$ and $B 2$. This type of localization could have a dramatic experimental
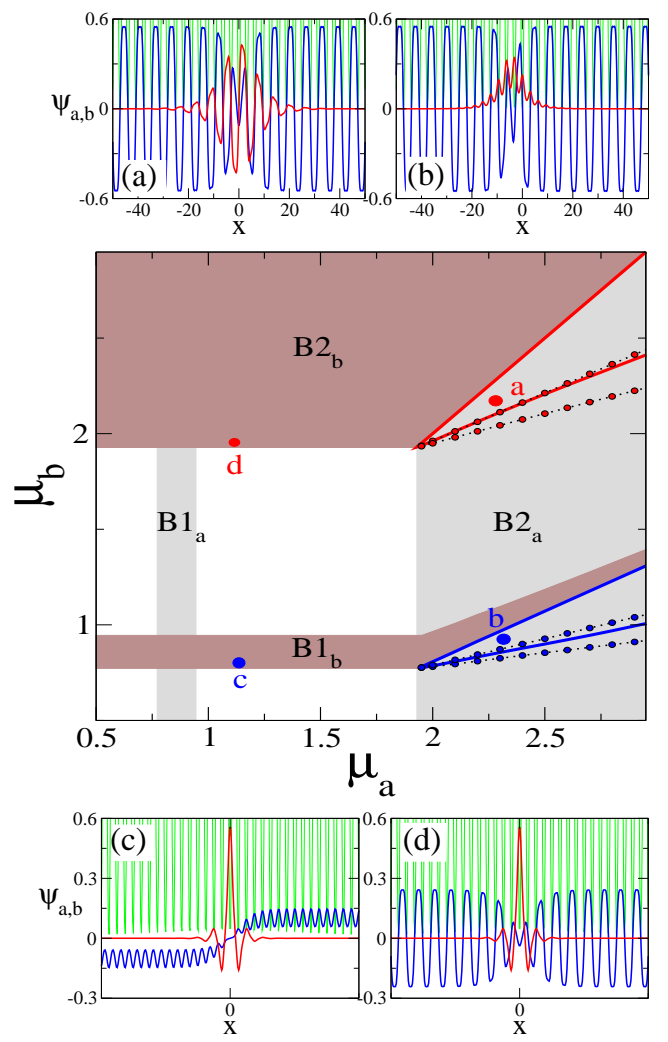

FIG. 3: Existence domain for the dark-bright atomic solitons $\left(V_{0}=2, g_{a a}=g_{b b}=g_{a b}=1\right)$. Shaded - two lowest Bloch bands $B 1_{a, b}$ and $B 2_{a, b}$ exhibited by the condensate components $|a\rangle$ and $|b\rangle$. Solid lines - borders of the existence domains in repulsive-repulsive regime derived from the linear waveguiding calculations (see text). Dotted lines - borders of the existence domains for the lattices shift $\theta=\pi / 4$. Top (bottom) panels: examples of the condensate wavefunctions in the repulsive-repulsive (attractive-repulsive) regime at the marked points of the existence domains.

manifestation, whereby formation of a bright soliton in one of the condensate components can be achieved by phase-imprinting onto the Bloch state of the complementary component in the spectral band. This effect can be observed when both components are located either at the same or the opposite band edges [Fig. 1(b)], but always in the normal diffraction regime. The localization of a single-component repulsive BEC in the form of a bright soliton is impossible in this regime. Therefore, although the dark-bright state can be dynamically stable [see Figs. 目 for a dark state imposed onto both

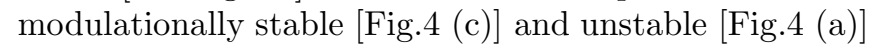
Bloch-wave backgrounds, decoupling of the condensate components leads to rapid spread of the bright localized state, proportional to its mean-field energy [Figs. 廿(b,d)].

Dark-bright solitons supported by effectively repulsive nonlinear interactions have been previously discussed in the context of nonlinear optics 18 and BEC in a harmonic potential [19]. The striking difference between 

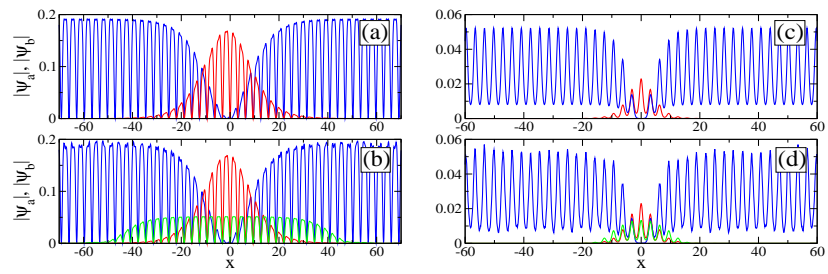

FIG. 4: Temporal evolution of a dark-bright localized state in the repulsive-repulsive regime with both components within (a,b) the second band, at $\mu_{a}=2.2, \mu_{b}=2.1$, and $(\mathrm{c}, \mathrm{d})$ the first band at $\mu_{a}=0.81, \mu_{b}=0.8$. Shown are (a,c) initial spatial density profiles and profiles of the coupled state and a spreading bright component decoupled from the dark one at (b) $t=1.35 \mathrm{mc}$ and (d) $t=2.7 \mathrm{mc}$. Note the slower expansion for the lower density state (c).

the dark-bright localized states in the optical lattice and dark-bright BEC solitons in a harmonic potential is that the bright component in the lattice is localized in the gaps of the induced band-gap structure. Therefore the multiple existence domains for the two-component state always lie between the cut-off line of the ground mode guided by a dark state and the corresponding edge of the induced band. In contrast with the case of bright-bright gap solitons, these existence domains shrink (dotted lines in Fig. 3) with the relative shift between the lattices $V_{a}(x)$ and $V_{b}(x)$, due to reduced effect of the induced periodic potential on the bands shift. Another drastic feature of the dark-bright states in the lattice is the energy difference between the states centered at the lattice minima and maxima, which inhibits mobility of the localized states across the lattice. As a result, the interaction of two dark-bright states with zero relative velocity (which, without the lattice, depends of the relative phase of the bright components [18]) is suppressed, and their spatial separation can be constant with time.

Repulsive-attractive regime [Fig. [(c)] can be accessed when $\tilde{g}_{a a}, \tilde{g}_{a b}<0, \tilde{g}_{b b}, \tilde{g}_{b a}>0,\left|\tilde{g}_{a b}\right|=\left|\tilde{g}_{b a}\right|$, and $\left|\tilde{g}_{a a}\right| \neq\left|\tilde{g}_{b b}\right|$. The BEC components should be located at different band edges, which is more difficult to arrange experimentally. However, in the absence of optical lattice, this regime could only be explored for a mixture of BECs with opposite signs of the scattering lengths. In this regime, the envelope equations (2) predict the existence of "normal" and "reverse" pairs of dark-bright solitons [20]. In the "normal" case, a dark soliton on the Bloch-wave background in the normal diffraction regime (e.g., at $\mu_{a} \geq \mu_{a}^{\{3\}}$ ) is coupled to a bright gap state in the anomalous diffraction regime (e.g., at $\left.\mu_{b} \geq \mu^{\{} 2\right\}_{b}$ ). Both components can exist independently as dynamically stable localized states, and the coupling is achieved for $\tilde{g}_{a b}^{2}<\left|\tilde{g}_{a a} \tilde{g}_{b b}\right|$. Typical examples of condensate wavefunctions in this regime are shown in Fig. 3(c,d). The bright localized component $|a\rangle$ exists in the entire gap, whereas the dark $|b\rangle$ component forms a coupled state only in the vicinity of the band edge. Although in the homogeneous case the two-component state is highly unstable with respect to the mutual displacement of its constituents [20], the optical lattice potential has a stabilizing effect on its dynamics. The "reverse" combination, when the dark state in the anomalous diffraction regime (effectively attractive condensate) is coupled to a bright state in the normal diffraction regime (effectively repulsive condensate) requires that $\tilde{g}_{a b}^{2}>4\left|\tilde{g}_{a a} \tilde{g}_{b b}\right|[20$, which is hard to achieve for realistic experimental conditions.

In conclusion, we have analyzed the two-component BEC in optical lattices and shown that spin-dependent lattices can be used to vary both the type and magnitude of nonlinear interactions without manipulating atomic scattering lengths. Using a simple envelope theory combined with the principles of linear waveguiding, we have demonstrated that localization of BEC in the form of gap solitons can occur both in the gaps and bands of the linear Bloch-wave spectrum. The formation of the dark-bright composite state is a striking effect that does not require accessing the spectral gaps, which makes it attractive for possible experimental observations.

We thank M. Oberthaler for many fruitful discussions.

[1] J.H. Denschlag et al., J. Phys. B 35, 3095 (2002).

[2] S. Peil et al., Phys. Rev. A 67, 051603 (2003).

[3] M. Jona-Lasinio et al., arXiv: cond-mat/0306210 (2003).

[4] E.A. Ostrovskaya and Yu.S. Kivshar, Phys. Rev. Lett. 90, 160407 (2003).

[5] For a review, see D. N. Christodoulides, F. Lederer, and Y. Silberberg, Nature 424, 817 (2003).

[6] H. Pu et al., Phys. Rev. A 67, 43605 (2003).

[7] See e.g., P.J. Louis et al., Phys. Rev. A 67, 013602 (2003), and references therein.

[8] B. Eiermann et al., Phys. Rev. Lett. 91, 060402 (2003).

[9] L. Fallani et al., arXiv: cond-mat/0303626 (2003).

[10] B. Eiermann, Th. Anker, M. Albeiz, M. Taglieber, and M. K. Oberthaler, submitted to Science.

[11] See, e.g., H. Pu and N.P. Bigelow, Phys. Rev. Lett. 80, 1130 (1998).

[12] O. Mandel et al., Phys. Rev. Lett. 91, 010407 (2003).

[13] B. Deconinck et al., J. Phys. A, 365431 (2003).

[14] N. A. Kostov et al., arXiv:cond-mat/0307156 (2003).

[15] A.A. Sukhorukov and Yu. S. Kivshar, Phys. Rev. Lett. 91 (2003); arXiv:nlin/0302058 (2003).

[16] G. Modugno et al., Phys. Rev. Lett. 89190404 (2002).

[17] A.V. Yulin and D.V. Skryabin, Phys. Rev. A 67, 023611 (2003).

[18] A.P. Sheppard and Yu.S. Kivshar, Phys. Rev. E 55, 4773 (1997).

[19] Th. Busch and J.R. Anglin, Phys. Rev. Lett. 87, 010401 (2001).

[20] Yu.S. Kivshar and G.P. Agrawal, Optical Soltions: From Fibers to Photonic Crystals (Academic, San Diego, 2003), Ch. 9, and references therein. 